\title{
Directed mutagenesis as a technique to study protein function: application to $\boldsymbol{\beta}$-lactamase
}

G. DALBADIE-McFARLAND,* A. D. RIGGS $\dagger$ and J. H. RICHARDS*

*Division of Chemistry and Chemical Engineering, California Institute of Technology, Pasadena, CA 91125, U.S.A., and †City of Hope Research Institute, Duarte, CA 91010, U.S.A.

The function of a protein follows uniquely from its threedimensional structure, which is unambiguously determined by the linear sequence of amino acids. Thus to undertake a systematic study of the relationship between protein structure and function, one would ideally like to be able to alter the structural gene in various ways to encode proteins with novel sequences, structures and functions. Various mutagenic strategies and methods have recently been developed that allow one to achieve these objectives. In one strategy, a particular amino acid sequence is specified and the functional properties of the resulting protein then determined; the most general method for accomplishing this approach is site-specific mutagenesis in which a synthetic oligonucleotide is used to direct very specific base changes to a specific site in the structural gene. In a complementary strategy, a particular function for the protein is specified and the amino acid sequence and structure of the protein that displays this property is then identified. The methods for accomplishing this objective involve various types of random mutagenesis, perhaps directed to a particular region of the structural gene, followed by phenotypic screening of the many resultant mutant proteins for any that possess the specified properties.

We have applied both these strategies to the enzyme $\beta$ lactamase whose structural gene (Sutcliffe, 1978) is a component of the plasmid pBR322. The active site contains a sequence $N \ldots \operatorname{Ser}(70)$-Thr-Phe-Lys $\ldots C$ in which the serine, threonine and lysine residues are conserved in enzymes from several different organisms (Ambler, 1980). Moreover, the serine residue at position 70 (Pratt \& Loosemore, 1978; Knott-Hunziker et al., 1979a,b; Loosemore et al., 1980; Cohen \& Pratt, 1980) is likely to function as a nucleophile in opening the $\beta$-lactam ring of penicillins to produce an acyl-enzyme intermediate (Fisher et al., 1980) which is subsequently hydrolysed to regenerate enzyme and release penicilloic acid which is inactive as an antibiotic.

In studies of this region of the enzyme we have generated all possible combinations of serine and threonine residues at positions 70 and 71 . Oligonucleotide-directed mutagenesis produced the two proteins with threonine at position 70 [the double mutant Ser-70 $\rightarrow$ Thr, Thr-71 $\rightarrow$ Ser (DalbadieMcFarland et al., 1982) and the single mutant Ser-70 $\rightarrow$ Thr]; both are catalytically inactive. A mutant that retained serine at position $70(\mathrm{Thr}-71 \rightarrow$ Ser) was obtained as a spontaneous revertant to ampicillin resistance of the double mutant and shows appreciable catalytic activity.

The procedure we have used for site-specific oligonucleotide-directed mutagenesis is outlined in Fig. 1 (Dalbadie-McFarland et al., 1982). The mutation is introduced into the structural gene by virtue of the base changes that have been incorporated into the synthetic oligonucleotide. Mutant colonies are identified, after transformation with plasmid and cloning in Escherichia coli as host, by the selective hybridization of the $32 \mathrm{P}$-labelled mutagenizing oligonucleotide to the mutant colonies to whose plasmid DNA the oligonucleotide binds with no base-pair mismatches. Binding of this oligonucleotide to the parental, wild-type colonies involves mismatches at those

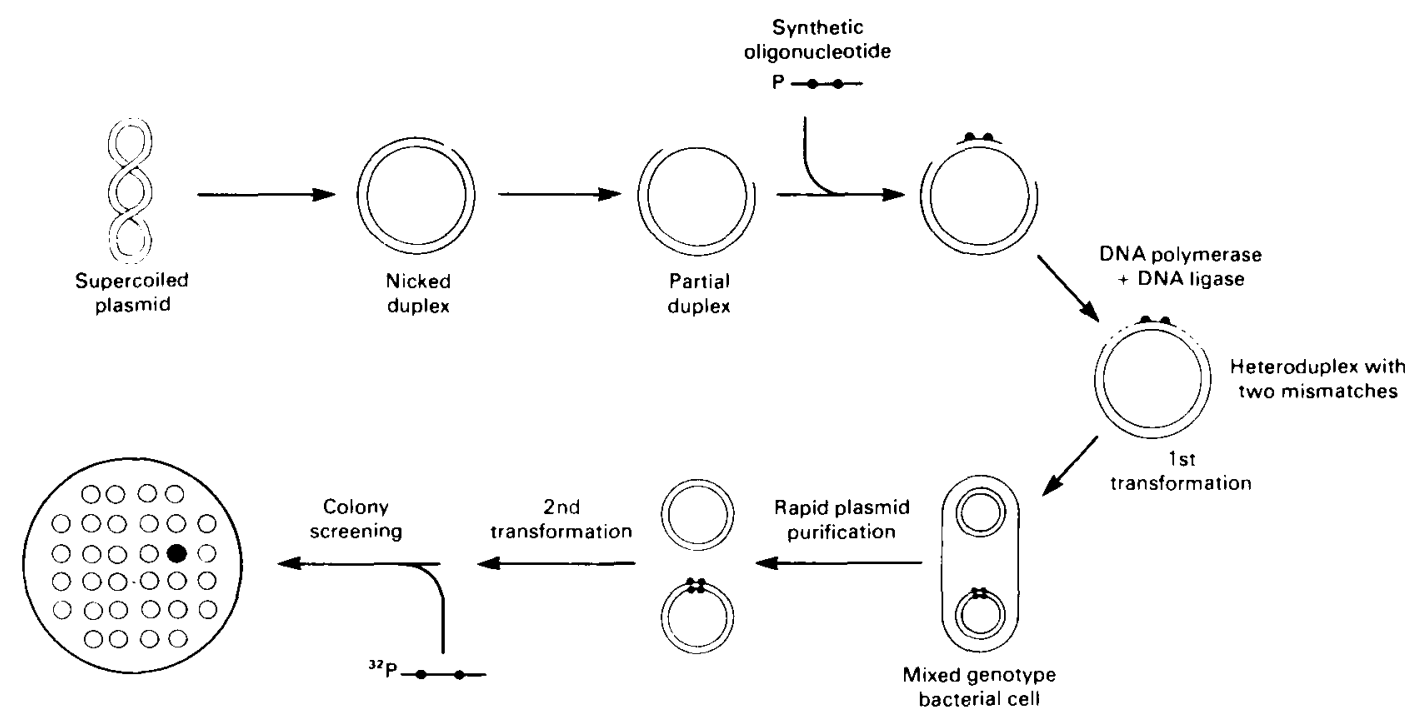

Fig. 1. Overall scheme for oligonucleotide-directed mutagenesis of double-stranded circular plasmid DNA

Supercoiled plasmid circles are nicked in one strand and rendered partially singlestranded by treatment with exonuclease. The gapped circles are hybridized with a homologous oligodeoxynucleotide carrying, by design, some mismatches. In vitro DNA synthesis, primed in part by the oligodeoxynucleotide, leads to heteroduplex plasmid circles. Molecular cloning and in vivo DNA replication generate homoduplexes, some of which have the DNA sequence of the primer oligodeoxynucleotide. Colony screening, with the same oligodeoxynucleotide labelled with ${ }^{32} \mathrm{P}$ as a hybridization probe, allows identification of the desired mutant colony regardless of its phenotype. 
positions that were altered in the mutant and is, accordingly, weaker. Thus, after washings at appropriate temperatures, the synthetic oligonucleodite remains bound to mutant colonies while it has been dissociated from parental colonies. In this procedure, since mutants are screened at the DNA level, one need not rely on phenotypic differences between mutant and parent; such screening makes this a powerfully general method for generating and identifying mutants with specified sequences.

In the alternative approach, one specifies a particular phenotype, in the present case lactamase activity, uses various methods of random mutagenesis to generate a large number of mutant proteins and then employs a screening procedure that depends on phenotype to identify colonies that produce a catalytically active enzyme.

\section{Site-directed mutants (Ser-70 $\rightarrow$ Ser and Ser-70 $\rightarrow$ Thr $)$}

We have previously reported the preparation of a double mutant of the residues in the catalytic site, specifically Ser$70 \rightarrow \mathrm{Thr}$; Thr-71 $\rightarrow$ Ser, in which the sequence of the residues

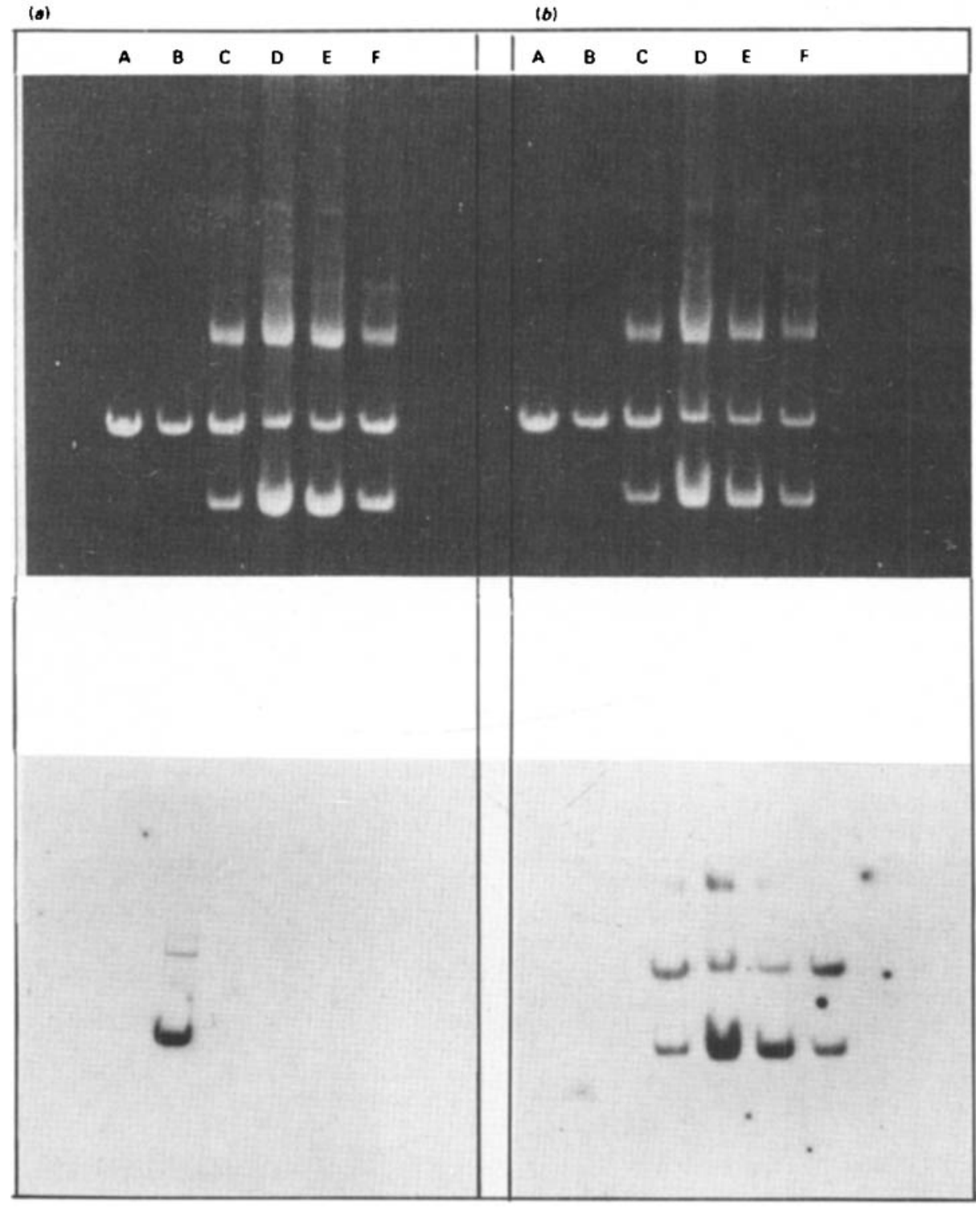

Fig. 2. Hybridizations of plasmid DNA from revertants to ampicillin resistance against two different oligonucleotide probes

(a) Ethidium bromide-stained gel (top) and autoradiogram (bottom) of the same gel after drying and hybridizing (Dalbadie-McFarland et al., 1982) to the oligomer 5'${ }^{32} \mathrm{P}-\mathrm{A}$ ATG ATG ACC TCT-3'. Hybridization conditions were as described previously (Dalbadie-McFarland et al., 1982) and washes were in $6 \times$ standard saline citrate (SSC) at $43^{\circ} \mathrm{C}$. (b) Ethidium bromide-stained gel (top) and autoradiogram (bottom) of the same gel after drying and hybridizing to the oligomer $5^{\prime}-32 \mathrm{P}-\mathrm{A}$ ATG ATG AGC TCT $-3^{\prime}$ as in $(a)$. Washes were in $6 \times \mathrm{SSC}$ at $50^{\circ} \mathrm{C}$. Lanes labelled A, EcoRI digest of pBR322 (-AGC ACT-); lanes labelled B, EcoRI digest of double mutant DNA (ACC TCT); lanes labelled C, D, E and F are partial EcoRI digests of plasmid DNA from revertants to ampicillin resistance. 
has been inverted. (Dalbadie-McFarland et al., 1982). This was accomplished by mutating the parental sequence in this region, 5'-AGC ACT-3', to 3'-ACC-TCT-3' (the asterisks denote the sites of base changes). By a similar approach, using the synthetic oligonucleotide 5'-A ATG ATG ACC ACT TTT-3', we have prepared the single mutant Ser$70 \rightarrow$ Thr. Both mutants with threonine in place of serine at position 70 show no detectable catalytic activity.

\section{Revertants}

In asking questions of the possible amino acid sequences that might be compatible with at least some degree of catalytic activity, one would like to screen phenotypically for such activity, without having any catalytically active mutants obscured by a high background of colonies that produce wild-type enzyme. To this end we employed a mutant that has two base changes relative to the wild-type and allowed random mutagenesis to occur at the normal rate characteristic of replication of plasmids in bacteria: a frequency of about 1 in $10^{6}$ for any given base pair. Thus we can expect a reasonable probability of examining all random mutants that differ by a single base change from the starting gene, but a very much lower probability of recovering wild-type enzyme from such a double mutant as this would require two base changes.

To produce such potentially active revertants, $E$. coli carrying the doubly mutant plasmid that encodes an inactive protein $(\mathrm{Ser}-70 \rightarrow \mathrm{Thr}$; $\mathrm{Thr}-71 \rightarrow \mathrm{Ser}$ ) was cultured in broth and then plated on Agar containing $10 \mathrm{mg} / \mathrm{l}$ of ampicillin. Seven colonies that had reverted to ampicillin resistance were isolated and, in six of these, the antibiotic resistance was carried on the plasmid. Plasmid DNA from four of these colonies was hybridized against two different oligonucleotide probes: 5'-A ATG ATG ACC TCT TTT-3' which matches the base sequence in the region of the active site of the double mutant, and 5'-ATG ATG AGC TCT TTT-3' which encodes the single mutant Thr-71 $\rightarrow$ Ser (i.e. an active site sequence of Ser-70, Ser-71). Fig. 2 shows the results of these hybridizations; the oligonucleotide for the double mutant binds specifically to the DNA of the double mutant under conditions where it dissociates from both wild-type and from all four of the putative Thr-71 $\rightarrow$ Ser mutants; the oligonucleotide that encodes the single $\mathrm{Thr}$ $71 \rightarrow$ Ser mutant binds to the four putative mutants under conditions where it dissociates from both the DNA of wildtype and double-mutant colonies.

Another mutant in this region, thiolactamase, with Ser$70 \rightarrow$ Cys (Sigal et al., 1982), is known to be catalytically active. It would not, however, have been expected to be generated by the procedure just outlined at a comparable frequency as either codon for cysteine (TGT or TGC) is at least two base changes away from the threonine codon $(A C C)$ in the doubly mutant gene from which the active revertant was prepared.

The Thr-71 $\rightarrow$ Ser mutant has about $30-50 \%$ of the catalytic activity of the wild-type enzyme, but is considerably less stable in the milieu of the periplasmic space than is wild-type. Enzyme can be efficiently produced by trans- ferring the structural gene to an overproducing plasmid where it is under the control of a hybrid trp-lac promoter (de Boer et al., 1983). Wild-type enzyme is abundantly produced by growing $E$. coli with this overproducing plasmid at $37^{\circ} \mathrm{C}$; the wild-type enzyme is reasonably stable in a crude extract under these conditions. In contrast, growth of $E$. coli with an overproducing plasmid for the Thr $-71 \rightarrow$ Ser mutant at $37^{\circ} \mathrm{C}$ yields virtually no active enzyme and cells must be grown at temperatures not above $30^{\circ} \mathrm{C}$ as the mutant protein is destroyed at higher temperatures. The lowered stability of the mutant seems due to its increased susceptibility to proteolysis rather than simple irreversible thermal denaturation.

\section{Second site revertants}

As an example of an approach to determine whether changes outside the region of the active site could restore catalytic activity to a protein that had threonine at position 70 , we hybridized the synthetic oligonucleotide 5 -A ATG ATG ACC ACT TTT-3' to the mutant plasmid that had long single-stranded regions produced by treatment with a restriction enzyme followed by exonuclease. The binding of the synthetic oligonucleotide will protect the region of the active site from mutation during subsequent in vitro replications that were carried out in the absence, in turn, of each of the four deoxynucleotide triphosphates to increase the frequency of mutagenesis at sites outside the region of residue 70 . No active second site revertants have yet been isolated by this procedure. While this negative result cannot yet be unambiguously interpreted, one possibility is that the presence of a primary nucleophile, either $-\mathrm{CH}_{2} \mathrm{OH}$ as in wild-type enzyme or $-\mathrm{CH}_{2} \mathrm{SH}$ as in thiolactamase, is an essential requirement for lactamase activity. The steric hinderance in a secondary alcohol such as threonine, $-\mathrm{CHCH}_{3} \mathrm{OH}$, might effectively prevent the nucleophilic attack on the substrate that is the first step in the catalytic mechanism.

Ambler, R. P. (1980) Phil. Trans. R. Soc. London Ser. B 289, 321 331

Cohen, S. A. \& Pratt, R. F. (1980) Biochemistry 19, 3995-4003

Dalbadie-McFarland, G., Cohen, L. W., Riggs, A. D., Morin, C., Itakura, K. \& Richards, J. H. (1982) Proc. Natl. Acad. Sci. U.S.A. 79, 6409-6413

de Boer, H. A., Comstock, L. J. \& Vasser, M. (1983) Proc. Natl. Acad. Sci. U.S.A. 80, 21-25

Fisher, J., Belasco, J. G., Khosla, S. \& Knowles, J. R. (1980) Biochemistry 19, 2895-2901

Knott-Hunziker, V., Waley, S. G., Orlek, B. S. \& Sammes, P. G. (1979a) FEBS Lett. 99, 59-61

Knott-Hunziker, V., Orlek, B. S., Sammes, P. G. \& Waley, S. G. (1979b) Biochem. J. 147, 365-367

Loosemore, M. J., Cohen, S. A. \& Pratt, R. F. (1980) Biochemistry 19, 3990-3995

Pratt, R. F. \& Loosemore, M. J. (1978) Proc. Natl. Acad. Sci. U.S.A. 75, 4145-4149

Sigal, I. S., Harwood, B. G. \& Arentzen, R. (1982) Proc. Natl. Acad. Sci. U.S.A. 79, 7157-7160

Sutcliffe, J. G. (1978) Proc. Natl. Acad. Sci. U.S.A. 75, 3737-3741 